\title{
19. Der Verleger Heinrich Beck
}

sechs Jahren kehrte er aus Anlass des 7o. Geburtstags seiner Großmutter Eugenie Rohmer zum ersten Mal wieder in seine Geburtsstadt Nördlingen zurück. Dort ließen ihn die alte Ringmauer, die hohen Wehrtürme und die steilen Hausgiebel glauben, er sei in einem Traumland. In der alten verwinkelten Druckerei zeigte der Obermaschinenmeister dem Knaben «eine sorgfältig gehütete ältere Nummer des «Nördlinger Anzeigeblatts`, die seine Geburtsanzeige enthielt. Das ganze Blatt war in goldener Farbe gedruckt.» ${ }^{2}$ Tief beeindruckt fuhr er in die Hauptstadt zurück.

In München besuchte Heinrich Beck das Königliche Maximilians-Gymnasium, das Maximilian II. 1849 als drittes Gymnasium der Residenzstadt gegründet hatte, um die neuhumanistische Ausbildung seiner begabten Untertanen zu gewährleisten. Im Sommer 1908 erwarb Heinrich Beck das Zeugnis der Reife. Wer gedacht hätte, dass der Sohn nun durch eine Buchhändlerlehre auf seine künftige Aufgabe vorbereitet würde, sah sich getäuscht. Sein Vater machte keine Anstalten, diese Familientradition fortzusetzen. Also wechselte der junge Mann nicht in das Kontor, sondern in den Hörsaal. Während des Wintersemesters 1908/og studierte er an der Ludwig-Maximilians-Universität Jurisprudenz, Nationalökonomie und Philosophie. Zu Ostern 1909 verbrachte er mehrere Monate in Paris. Im Herbst 1909 bezog er die Friedrich-Wilhelms-Universität in Berlin, wo er bis Sommer 1910 Philosophie, Geschichte, Kunstgeschichte und Deutsche Philologie hörte. Vom Herbst 1910 bis Sommer 1913 widmete er sich denselben Studienfächern an der Universität Leipzig. ${ }^{3}$ Sein akademischer Lehrer war der Philosoph Johannes Volkelt, der mit Oskar Beck seit einem gemeinsamen Kuraufenthalt im Fichtelgebirge befreundet war. C.H.Beck verlegte zahlreiche seiner Schriften zur Ästhetik. ${ }^{4}$ Heinrich Beck hörte zudem bei Georg Witkowski und Albert Köster über die Geschichte der deutschen Literatur, bei Wilhelm Wundt über Hauptprobleme der Völkerpsychologie, bei Karl 
Lambrecht über Kultur- und Universalgeschichte, bei August Schmarsow und Martin Wackernagel über die Kunstgeschichte der Renaissance und des Barock und bei Eduard Spranger über die Philosophie der Geschichte. Mit Spranger stand er auch später in Verbindung, konnte ihn aber nicht als Verlagsautor gewinnen. ${ }^{5}$ Er war nur Beiträger zur Festschrift von Volkelt, die 1918 zum 70. Geburtstag des Philosophen bei C.H.Beck erschien.

\section{Im Ersten Weltkrieg}

Im Herbst 1913 trat Heinrich Beck in München als Wehrpflichtiger mit Abitur das sogenannte Einjährig-Freiwilligenjahr beim Bayerischen 7. Feldartillerie-Regiment an. Dann brach der Erste Weltkrieg aus. Von Beginn an trug Heinrich Beck den grauen Rock. Die erste Feindberührung hatte er am 10. August 1914 in Lothringen. Die anfängliche Euphorie wich bald der Ernüchterung, als der Unteroffizier erkennen musste, dass der SchlieffenPlan gescheitert und die Einkesselung der französischen Armee misslungen war. Aus dem Bewegungskrieg wurde ein Stellungskrieg. Vier lange Jahre versah Heinrich Beck seinen Dienst an der Westfront, wurde am 1. Januar 1915 zum Vizewachtmeister und am 26. April 1915 zum Leutnant der Reserve befördert. Als Artillerieoffizier befehligte er die intensiven Bombardements, die das Bild eines anonymen und entpersonalisierten Krieges prägten. Er nahm an Angriffskämpfen und Abwehrschlachten bei St. Quentin, Arras und Fort Thiaumont, zwischen Maas und Mosel und an der Aisne teil. An der Somme wurde er am 11. Oktober 1915 durch einen französischen Granatsplitter am linken Unterarm verwundet und musste mehrere Wochen im Lazarett versorgt werden. Die kurze Unterbrechung, die sich anschloss, nutzte er, um wieder seinen philosophischen Studien nachzugehen. Am 16. Februar 1916, 5 Uhr 25, wurde er neuerlich ins Feld abkommandiert. Stellungskämpfe im Artois folgten. In einem Offizierslehrgang vertiefte er seine artilleristischen Kenntnisse. Ende des Jahres erkrankte er im nordfranzösischen Metz-en-Couture an Gelbsucht. Wieder lag er im Lazarett. Ein zweiwöchiger Erholungsurlaub schloss sich an, dann lag er erneut in seiner Batterie, die an der Westfront feindliche Stellungen beschoss. In die «Blutmühle» von Verdun wurde er im Oktober 1917 geschickt, wo er bis Juni 1918 seinen Dienst leistete. Im Sommer nahm er an der letzten deutschen Offensive bei Reims und in der Champagne teil und wurde zum Oberleutnant der Reserve befördert. Während der Hunderttageoffensive der Alliierten half er, die deutsche Front zu sta- 
blisieren. Am 11. November 1918, am Tag des Waffenstillstands, stand er an der Maas. Am nächsten Tag trat er den mühsamen Rückmarsch in die Heimat an. Zu Weihnachten traf er in München ein, dekoriert mit dem preußischen Eisernen Kreuz I. und II. Klasse und dem bayerischen MilitärVerdienst-Orden IV. Klasse mit Schwertern und mit Krone und Schwertern. $^{6}$

In seinem Tornister trug er die handschriftliche Abschlussbeurteilung seines Hauptmanns und Abteilungskommandeurs. Darin stand zu lesen, dass der Oberleutnant Heinrich Beck «in jeder Hinsicht Vorzügliches geleistet» habe. «Zäh und hingebend hat er jegliche Aufgabe auch in schwierigster Lage gelöst vermöge seiner Besonnenheit und Kaltblütigkeit, die ihn selbst im heftigsten feindlichen Feuer nie verlassen hat.» Insbesondere habe er sich als Batterieführer in der Angriffsschlacht am 15. Juli 1918 und in den folgenden Abwehrkämpfen an der Somme bewährt. Sehr gute artilleristische Kenntnisse wurden ihm bescheinigt. «Seinen Untergebenen war er stets ein Vorbild treuester Gewissenhaftigkeit und Pflichterfüllung.» Er habe ihr volles Vertrauen besessen, «das er sich durch unermüdliche Fürsorge für sie erworben hatte. Unbedingter Gehorsam seiner Untergebenen» sei «ihm sicher». Vorgesetzten gegenüber habe er «stets ein sehr taktvolles Benehmen an den Tag» gelegt. «Außerdienstlich war sein Auftreten stets einwandfrei. Äußerlich etwas zurückhaltend, war er ein treuer, beliebter Kamerad.» Der Regimentskommandeur, Major Schwarzenberger, pflichtete bei: «Sehr unerschrocken und kaltblütig, von bestem Einfluss auf seine Leute. Hat als Batterieführer in schwierigsten Lagen Ausgezeichnetes geleistet.» ${ }^{7}$

Der Krieg aber hatte Spuren an Körper und Seele hinterlassen. In den Schützengräben war die bürgerliche Welt zusammengebrochen, und das «Fronterlebnis» warf die Frage nach dem Sinn von Tod und Leben auf. Auch der privilegierte Offizier, der eine bessere Verpflegung erhielt und in sichereren Bunkern Unterschlupf fand, teilte mit dem gemeinen Soldaten einen «tiefverwurzelten Überlebenswillen» und den «unzerstörbaren Wunsch, trotz des großen Mordens in die Heimat zurückkehren zu können». ${ }^{8}$ Dort angekommen, musste inmitten des politischen Umsturzes die militärische Niederlage verarbeitet werden. Körperliche Probleme kamen hinzu. Seit Sommer 1917 litt Heinrich Beck an einem «chronischen Darmkatarrh», der seine «Fähigkeit zur Dienstleistung teilweise schwer beeinträchtigte», wie auch das militärärztliche Zeugnis von Januar 1919 bestätigte, das den Zusammenhang mit dem Felddienst und eine «Kriegsdienstbeschädigung» attestierte. ${ }^{9}$ Dieses Leiden begleitete Heinrich Beck sein ganzes Leben, 


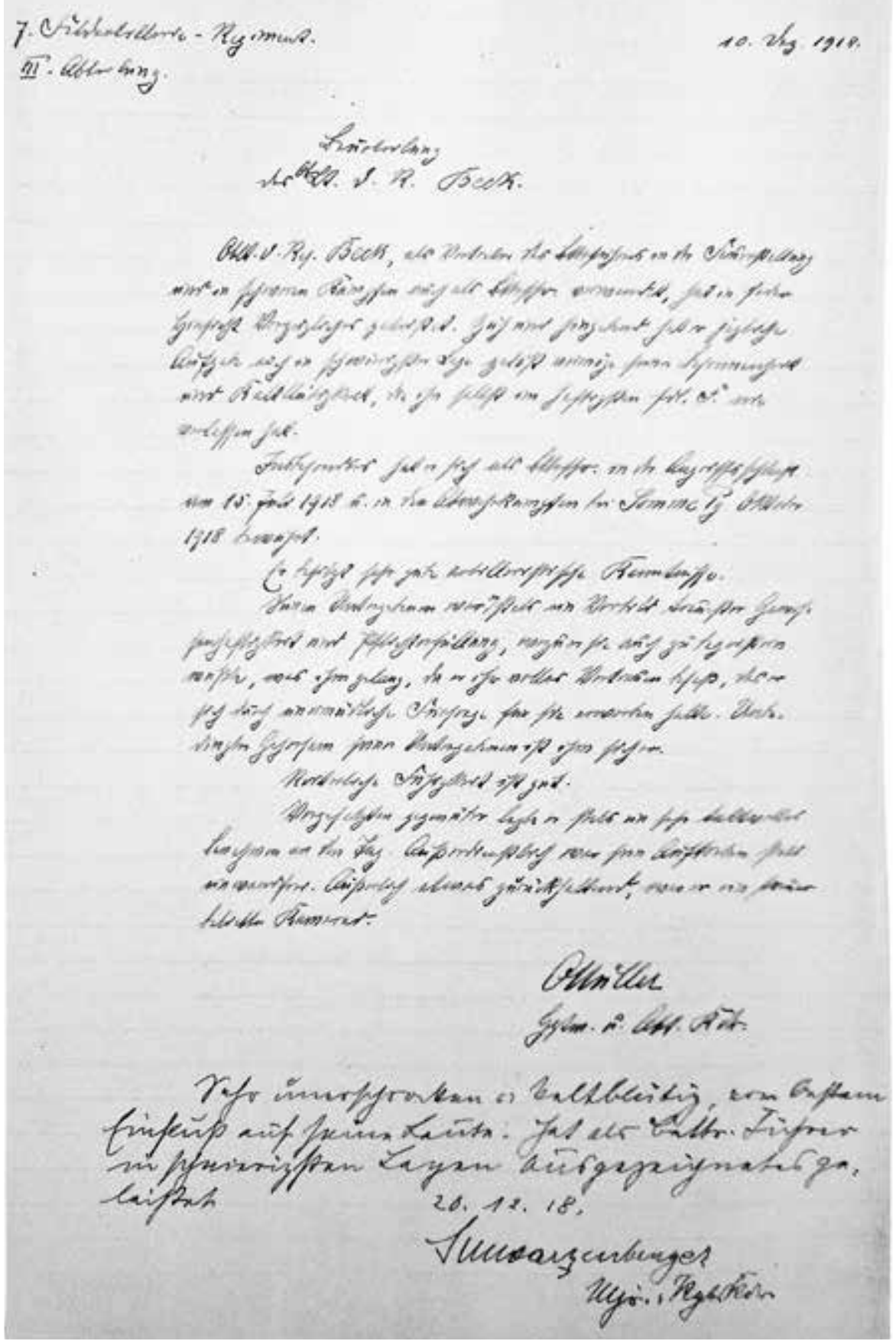

Positive Abschlussbeurteilung des Oberleutnants Heinrich Beck durch seine Vorgesetzten 
und penibel verzeichnete er in seinem Tagebuch seine fortdauernde gesundheitliche Beeinträchtigung. Versorgungsansprüche erhob Heinrich Beck allerdings nicht, sondern trat im Januar 1919 mit 29 Jahren völlig unvorbereitet «seine verlegerische Laufbahn» an. Sein Vater war «schwer krank», so dass dieser ihn noch nicht einmal «in seinem Beruf anzuleiten» vermochte. Jetzt rächte es sich, dass ihm eine einschlägige Ausbildung fehlte, um die notwendige «Reorganisation der altväterlichen Buchführung und Arbeitsteilung» durchzuführen. ${ }^{10}$ Heinrich Beck fand Anleitung und Hilfe bei den Verlagsmitarbeitern August Albers und Walther EggertWindegg, die uneigennützig halfen, das Unternehmen durch Revolution und Inflation hindurchzusteuern und das Programm zu erweitern. Kaufmännischen Rat gab der energische Prokurist Karl Schröpel, der seine Lehre in Nördlingen absolviert hatte und 1909 in den Verlag eingetreten war. ${ }^{11}$ Der junge Verleger musste sozusagen im eigenen Verlag in die Lehre gehen.

Von der Universität zum Verlag

Heinrich Beck wollte indes nicht nur Unternehmer sein. Der studierte Philosoph suchte Erklärungen für die Krise der Gegenwart, Antworten auf die politischen und sozialen Konvulsionen, Ideen für die Zukunft. Er entschloss sich, seine Dissertation über den französischen Denker Henri Bergson abzuschließen, der in Auseinandersetzung mit den zeitgenössischen Naturwissenschaften eine metaphysische Lebensphilosophie entwickelt hatte, die der Suche nach dem élan vital gewidmet war. Anfang Februar 1921 legte Heinrich Beck der Philosophischen Fakultät der Universität Leipzig seine in München niedergeschriebene Doktorarbeit vor. Darin behandelte er in zehn Kapiteln «Henri Bergsons Erkenntnistheorie». Die Untersuchung war konventionell. Der Autor löste Bergsons Erkenntnistheorie «aus ihrer Verflechtung mit biologischen, psychologischen und metaphysischen Gedankenverbindungen», verglich sie «mit der modernen deutschen Erkenntnistheorie» und unterzog den Denker der Kritik. ${ }^{12}$ Die Referenten waren nur bedingt angetan. Heinrich Becks Lehrer Johannes Volkelt bescheinigte, dass der Kandidat sich eine sehr schwierige Aufgabe gestellt habe, da Bergson die im eigentlichen Sinn erkenntnistheoretische Fragestellung nicht kenne. «Jenem Eingebettetsein der erkenntnistheoretischen Gedanken in Bergsons gesamter Philosophie hat der Verfasser dadurch Rechnung getragen, dass er kapitelweise die wichtigsten Lehr- 
stücke Bergsons vornimmt und sie nach ihrem erkenntnistheoretischen Einschlag untersucht. Regelmäßig gibt er zuerst eine Darstellung des Bergson'schen Gedankenzusammenhanges und lässt dann kritische Betrachtungen, oft recht umfangreicher Art, folgen. Jede Darstellung ist zutreffend, hebt die Grundgedanken hervor und zeugt allenthalben von langem, gründlichem Studium Bergsons.» Jedoch sei die Eigenart der Bergson'schen Philosophie nicht deutlich zum Ausdruck gebracht worden. «Überhaupt entbehrt die ganze Art des Verfassers, den ich als einen, in betontem Sinne ‘feinen> Kopf kenne, des Zugreifenden, fest Anfassenden.» Der Doktorand hatte sich zu eng an den Text geklammert und keine selbständigen Positionen entwickelt: «Es wäre durchaus am Platz, wenn der Verfasser seine eigenen erkenntnistheoretischen Anschauungen an angemessenem Orte kurz und bestimmt darlegte. Vor einer etwaigen ins Auge gefassten Drucklegung wäre dies nachzuholen und die Arbeit überhaupt in der Richtung zu verbessern, dass den kritischen Ausführungen eine deutlichere Sprache gegeben würde.» ${ }^{13}$

Der Kandidat wurde zur mündlichen Prüfung zugelassen und bestand am 10. Mai 1921 das examen rigorosum in den Fächern Kunstgeschichte, Deutsch und Philosophie. Der Dekan, der Klassische Philologe Richard Heinze, dekretierte am 2 1. Mai, die Annahme der Arbeit mit der Note III sei beschlossen, da der Kandidat erklärt habe, «eine tiefer gehende Umarbeit nicht vornehmen zu wollen». ${ }^{14}$ Die Verlagsbuchhandlung verlangte Heinrich Becks ganze Aufmerksamkeit. Wissenschaft als Beruf war keine Option. Heinrich Beck erwarb den Titel und verzichtete auf die Revision. Von der Dissertation ist kein Exemplar mehr vorhanden. In der Bergson-Forschung hat sie keinen Nachhall gefunden.

Doch bot der Verlag eine sichere Zukunft? Die schon von den Zeitgenossen beobachtete und kommentierte Entfaltung der «Massenkultur», der Aufstieg der «Massenmedien», der Presse, des Films und des Rundfunks, gingen mit einer vermeintlichen «Bücherkrise» einher, die der Verleger Samuel Fischer 1926 diagnostizierte. Das «gute Buch», das «an die Seele» rühre, finde immer weniger Leser. Das Kulturgut Buch werde «zu den entbehrlichsten Gegenständen des täglichen Lebens»: «Man treibt Sport, man tanzt, man verbringt die Abendstunden am Radioapparat, im Kino» und finde keine Zeit, ein Buch zu lesen. Die Gründe für diese Entwicklung waren rasch benannt: «Der verlorene Krieg und die amerikanische Welle haben unsere Lebensauffassung umgeformt, unseren Geschmack verändert.» Die eigentliche Ursache lag aber woanders: «Es scheint, als ob die aus dem Zusammenbruch hervorgegangene bürger- 
liche Gesellschaft, die vor dem Krieg die wirtschaftliche und kulturelle Führung hatte, in Auflösung begriffen ist.» ${ }^{15}$

Auch C.H.Beck war von dem «Geltungsverlust eines bürgerlich-individualistischen Bildungs- und Kulturbegriffs» ${ }^{16}$ betroffen und reagierte darauf mit einer dreifachen Strategie. Zum einen wurde durch die Initiative von August Albers und mit der Billigung Heinrich Becks das Programmsegment Kulturphilosophie entwickelt. Zum anderen antworteten Heinrich Beck und seine engsten Mitarbeiter auf die Pluralisierung der gesellschaftlichen Wertvorstellungen mit der weiteren Diversifizierung des Programms. Drittens stärkten sie die geisteswissenschaftlichen Schwerpunkte, die Altertumskunde, die Philosophie, die Geschichte und die Germanistik, auf Kosten der Jurisprudenz und der Theologie. Juristische Titel aus Bayern gingen in der preußisch dominierten Republik ohnehin schlecht. Und der Agnostiker Heinrich Beck glaubte nicht, mit theologischen Publikationen den Herausforderungen der Zeit begegnen zu können. Er erachtete es daher als sinnlos, dieses Gebiet weiter auszubauen. Sein Desinteresse kompensierte er durch die Förderung anderer Gebiete wie Religionswissenschaft und Religionsphilosophie.

Zunächst jedoch gaben die wirtschaftliche und die politische Entwicklung Anlass zur Sorge. Sein Vater Oskar Beck hatte bereits Anfang April 1922 geklagt, dass «alles so schwierig geworden» sei. Die «Kontorangestellten erhielten fortwährend Gehaltserhöhungen», und dennoch herrsche «eine kleine Palastrevolution». Die fortschreitende Geldentwertung ließ ihn voller Existenzängste in die Zukunft blicken. «Wer kann wissen, wie es in einem Jahr in Deutschland aussieht, ob wir bis dahin nicht den Lumpenkönig haben, der uns alle zugrunde richten wird?», fragte er verstört. C.H.Beck verlor Autoren und Mitarbeiter: Will Vesper wechselte zu Haessel in Leipzig und dann zu Bertelsmann in Gütersloh, und der Prokurist Hans Trüdinger wurde Teilhaber einer Kommanditgesellschaft für den Verkauf landwirtschaftlicher Maschinen. ${ }^{17}$ Papierknappheit erschwerte die Neuproduktion, und die Geldentwertung vernichtete die Gewinne. Der Buchhandel führte in der Inflationszeit ein neues Verkaufssystem ein: Rechnungen wurden in «Grundzahlen» erstellt, für die in bestimmten Abständen, 1923 dann fast täglich, ein Multiplikator, die sogenannte Schlüsselzahl, festgesetzt wurde. Der Buchpreis richtete sich nach der mit der Schlüsselzahl multiplizierten Grundzahl, und auf derselben Grundlage kalkulierte man die Rechnungen an die Verleger und die Honorare für die Autoren. ${ }^{18}$ Doch die Preise stiegen unablässig: Für Bieses Literaturgeschichte musste man in der zweiten Jahreshälfte $19239^{1 / 2}$ Billionen Mark 
bezahlen. Der Autor erhielt für eine Auflage von 4400 Exemplaren 200 Billionen überwiesen, die nach der Währungsreform gerade noch 15 Rentenmark wert waren. ${ }^{19}$ Doch zumindest die Druckerei in Nördlingen profitierte von der galoppierenden Inflation: Weil die Kapazität der Reichsdruckerei schon lange nicht mehr ausreichte, um immer mehr Banknoten mit höheren Werten herzustellen, sprang C.H.Beck wie so manche andere private Druckerei ein. In zwei Arbeitsschichten wurde, kontrolliert von einem groBen Stab von Aufsichtspersonen, der steigende Bedarf an Papiergeld befriedigt. Heinrich Beck selbst reiste regelmäßig des Nachts nach Berlin, um die neuen Klischees für Nördlingen abzuholen, die stetig höhere Nennbeträge auswiesen. ${ }^{20}$

\section{Programmentwicklung}

Mit solchen Maßnahmen war allerdings keine langfristige Verlagspolitik zu machen. «Der Wechsel der Generationen» bedingte, wie Heinrich Beck später schrieb, dass man im Verlag «tastend» eine Programmstruktur zu entwickeln versuchte. Noch mischte sich der Seniorchef in Entscheidungen ein, löste «in tief pessimistischer Stimmung» Verlagsverträge auf und sträubte sich gegen Veränderungen. ${ }^{21}$ In dieser Situation ergriff Heinrich Beck die Initiative, die sich ihm durch Oswald Spengler bot. Mit seiner Hilfe überwand Heinrich Beck die von seinem Vater vorgegebene Ausrichtung der Verlagspolitik entlang der neuhumanistisch-idealistischen Bildungstradition des 19. Jahrhunderts. Nach Oskar Becks Tod am 22. Januar 1924, konnte Heinrich Beck ein Programm realisieren, das durch das Medium Buch Antworten auf die «Krise der Moderne» zu geben suchte und die politischen, sozialen und geistigen Bewegungen der Zeit beeinflussen wollte. Er verstand sich selbstbewusst als «kultureller Verleger», ${ }^{22}$ der mit seinem Programm nicht mehr nationalliberale Ideen, sondern kultur- und zivilisationskritische Konzepte verbreitete. «Volksbildung» und «Leserlenkung» schrieb sich Heinrich Beck im Dauerstreit um die kulturellen und politischen Leitbilder in der Weimarer Republik auf seine Fahnen. ${ }^{23} \mathrm{Er}$ sammelte sicher nicht wie S. Fischer «die ganze Moderne in seinem Verlag», ${ }^{24}$ aber immerhin wurde C.H.Beck unter seiner Ägide zum Verlag der «Weimarer Trias〉 Oswald Spengler, Albert Schweitzer und Egon Friedell. Heinrich Beck stieß zur rechten Zeit auf die richtigen Autoren - oder genauer: Er profitierte davon, dass andere Verleger diese Autoren vergrault oder verärgert hatten und sie durch die Vermittlung der Lektoren zu 
C.H.Beck kamen. Die drei Autoren vertraten divergierende kulturphilosophische Vorstellungen und erschienen dennoch in ein und demselben Verlag: Pluralismus und Dissens kennzeichneten die Weimarer Kultur und ebenso die Programmpolitik des ambitionierten jungen Kulturverlegers Heinrich Beck.

Aber Heinrich Beck verstand rasch, dass mit Spengler, Schweitzer und Friedell allein kein Programm zu machen war. C.H.Beck konnte und sollte nicht in einen reinen Kulturverlag verwandelt werden, sondern blieb ein Universalverlag. Die Altertumswissenschaften bildeten nach wie vor einen Schwerpunkt. Fachvertreter der altertumskundlichen Disziplinen wollten bei C.H.Beck publizieren und bedauerten, wenn sie nicht zum Zuge kamen. Der Altorientalist Alfred Jeremias, der sich darüber beklagte, dass die Drucklegung seines überarbeiteten «Handbuchs der altorientalischen Geisteskultur» bei Hinrichs schrecklich mühsam sei, gab sich gewiss: «Wenn Beck das Buch genommen hätte, wäre ich wohl längst beim Druck.» ${ }^{25}$ Das Buch erschien 1929 bei Walter de Gruyter.

Das altertumswissenschaftliche Flaggschiff der Beck'schen Verlagsbuchhandlung, das «Handbuch der klassischen Altertumswissenschaft», wurde von zahlreichen weiteren Publikationen begleitet. $\mathrm{Zu}$ diesen zählte auch die große Habilitationsschrift des jungen Althistorikers Helmut Berve. «Das Alexanderreich auf prosopographischer Grundlage», in der die Struktur dieses Reiches auf der Grundlage von über 800 «Personengeschichten» zu erfassen versucht wurde; sie erschien $19^{2} 6$ in zwei Bänden bei C.H.Beck.

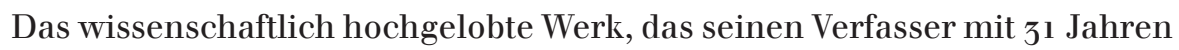
auf den renommierten Lehrstuhl für Alte Geschichte an der Universität Leipzig katapultierte, ${ }^{26}$ war indes ein Ladenhüter. Am 3. Oktober 1928 waren gerade einmal 203 Exemplare verkauft. Das gelehrte Werk erreichte nur einen denkbar kleinen Kreis von Spezialisten. «Es ist betrüblich, dass bei einem Werk dieser Art, das literarisch einen so unbestrittenen Erfolg errungen hat, die Absatzziffer doch eine so begrenzte ist und dass der Verleger schlechterdings nicht imstande ist, mit den Einnahmen die Kosten der Drucklegung zu bestreiten. Selbst der übliche Zuschuss der Notgemeinschaft reicht zu der Deckung des Fehlbetrags noch nicht aus», ließ Heinrich Beck den Ordinarius wissen. Dieser sollte seine einflussreiche Stellung nutzen, um im Kreis seiner Schüler für den Absatz des Buches zu sorgen. Sonst müsse sich der Verfasser, wie vertraglich vereinbart, an den Druckkosten beteiligen. ${ }^{27}$ Die Korrespondenz zeigt die zunehmende Abhängigkeit gerade junger Wissenschaftler von den Verlegern. ${ }^{28}$

Trotz der schwierigen Bedingungen der Nachkriegszeit erklärte sich 
C.H.Beck bereit, den bereits erwähnten «Kommentar zum Neuen Testament aus Talmud und Midrasch» herauszubringen, den zu veröffentlichen Hermann L. Strack, der dem Verlag vier Jahrzehnte lang verbunden war, wohl noch Oskar Beck überzeugt hatte. Der Vertrag datiert von Ende Juli 1920. Wissenschaftlich war das Unternehmen höchst innovativ, finanziell aber riskant. Deshalb wurde eine Sonderfinanzierung vereinbart: Strack verpflichtete sich, die Herstellung durch einen Zuschuss von 56 ooo Mark zu unterstützen, der auch für die Neubearbeitung seiner «Einleitung in Talmud und Midrasch» verwendet werden sollte. Es wurde zudem ein Sonderkonto geführt, auf dem einerseits die Einnahmen durch weitere $\mathrm{Zu}$ schüsse und den Verkauf, andererseits die Ausgaben durch Herstellung, Werbung und Geschäftsunkosten verbucht wurden. Der erste Band mit Erläuterungen zum Matthäusevangelium erschien 1922. Das maßgeblich von dem evangelischen Pfarrer und Judaisten Paul Billerbeck bearbeitete Werk ist bis heute lieferbar. ${ }^{29}$

Die Bemühungen um die traditionellen Altertumswissenschaften und ihre Nachbardisziplinen wurden in der akademischen Zunft positiv wahrgenommen und führten zur Anerkennung im wissenschaftlichen Feld: $193^{2}$ wurde C.H.Beck der Kommissionsverlag der Bayerischen Akademie der Wissenschaften, der die Herstellung und den Vertrieb ihrer zuvor im Selbstverlag herausgebrachten Abhandlungen und Jahrbücher übernahm. Deshalb erschienen ab 1937 bei C.H.Beck die von der Akademie betreuten «Gesammelten Werke Johannes Keplers», und 1940 wurde Heinrich Beck die Verdienstmedaille der Akademie «Bene merenti» verliehen.

Dennoch sollte die historistische Richtung das altertumswissenschaftliche Verlagsprogramm nicht exklusiv dominieren. Auch hier wurde nach der Verbindung von Wissenschaft und Leben gefahndet. So kommt es nicht von ungefähr, dass bei C.H.Beck in den zwanziger Jahren der Schweizer Mythenforscher und Theoretiker des Mutterrechts, Johann Jakob Bachofen, wiederentdeckt wurde, dessen Werke damals nicht in einer Gesamtausgabe greifbar waren. Den Grundgedanken, dass die Gynaikokratie mit Mutterrecht, Gütergemeinschaft, Naturverbundenheit und sexueller Promiskuität am Anfang der Menschheitsgeschichte gestanden habe, hatte man bereits um die Jahrhundertwende in der Schwabinger Kosmikerszene um Ludwig Klages attraktiv gefunden. Doch in den zwanziger Jahren kam es zu einer Bachofen-Renaissance, für die Manfred Schröter und Alfred Baeumler verantwortlich waren. Eine antimoderne Lektüre machte Bachofen zum gegenaufklärerischen Geschichtsphilosophen, der in eine Reihe mit Spengler gestellt wurde. Schröter edierte Bachofens «Autobio- 
graphischen Rückblick» auf handgeschöpftem Büttenpapier in 150 numerierten Exemplaren für die Rupprecht-Presse (1923) und gab für C.H.Beck zwei Bachofen-Texte heraus, «Oknos der Seilflechter. Ein Grabbild. Erlösungsgedanken antiker Göttersymbolik» (1923) und «Der Mythus von Orient und Occident. Eine Metaphysik der alten Welt» (1926). ${ }^{30}$

Die Geschichte des Oknos erzählte, wie Vater und Sohn in die Unterwelt hinabsteigen, wo vom Totengericht Verurteilte Seile flechten, die von Eseln aufgefressen werden. Der Klappentext pries Bachofen als «das wichtigste Mittelglied zwischen der älteren Romantik und Friedrich Nietzsche»; das Buch begann mit einem programmatischen Zitat aus Stefan Georges «Das Jahr der Seele», und Schröter forderte in seiner 50-seitigen Einleitung, die mit Bachofens Hilfe zu vollziehende Hinwendung zur Religionsgeschichte müsse sowohl «gegen die Beschränkung der exakten bloßen Wissenschaftlichkeit» als auch «gegen die Schwarmgeisterei und Schrankenlosigkeit des Sektenwesens und kultartiger Verehrung» gerichtet sein. Er polemisierte gegen die «zerstörende Kraft der Wissenschaft» und pries den genialen und populären Deuter antiker Mythen als Vorbild «inneren Verstehens und Miterlebens» und «der theoretischen Verknüpfung von Intuition und Wissensmaterial». Die Mutter, «das Ursymbol des ganzen Daseins», habe Bachofen «innig schwermütig vertieft zum seelischen Begegnungsort von wehmutsvoll begreifenden und von getröstet hoffenden geheimen Regungen des Gott- und Weltgefühls der Kreatur». ${ }^{31}$

Für die Anthologie «Mythus von Orient und Occident», die vor allem aus dem «Mutterrecht» zitierte, hatte der Philosoph Alfred Baeumler, der im «Dritten Reich» die Rasse als Grundbegriff der Erziehungswissenschaft entdecken sollte, die Einleitung verfasst. Damals war er noch ein notleidender Privatdozent an der Technischen Universität Dresden und auf die finanzielle Unterstützung durch Heinrich Beck angewiesen, der ihm Vorschüsse zahlte. ${ }^{32}$ Auf fast 300 Seiten wurde Bachofens Apotheose vollzogen. Sein Werk repräsentierte eine Wahrheit jenseits der traditionellen Wissenschaft. In der «Mythologe der Romantik» wurde von Baeumler mit Nietzsche verglichen und gegen die Klassische Philologie Karl Lachmanns und Ulrich von Wilamowitz-Moellendorffs in Stellung gebracht. Deren «rein ethisch-ästhetische Tradition» sei obsolet. Baeumler forderte eine Wissenschaft vom Altertum, die nicht vom «Verstande», sondern von der «Einbildungskraft» und «der schauenden Phantasie» geprägt werde. ${ }^{33}$ Die Auswahl insgesamt, aber vor allem Baeumlers Vorwort beeinflussten die Bachofen-Rezeption im wissenschaftlichen und literarischen Milieu. 4o Besprechungen erschienen innerhalb von zwei Jahren. ${ }^{34}$ Der Althistoriker 
Helmut Berve begrüßte «Mythus von Orient und Occident» im «Gnomon», auch wenn ihm Baeumlers Hinführung zu ausufernd und zu wenig altertumswissenschaftlich schien, ${ }^{35}$ der Soziologe Ernst Karl Winter sprach bereits 1928 von einer Bachofen-Renaissance, ${ }^{36}$ und Heidegger empfahl Bachofens Selbstbiographie seiner Freundin Elisabeth Blochmann zur Lektüre. ${ }^{37}$ Nur Thomas Mann distanzierte sich von der «Nachtschwärmerei» und dem «revolutionären Obskurantismus», ließ sich aber dennoch von dem Buch bei der Konzeption seiner «Josephs»-Tetralogie beeinflussen. ${ }^{38} \mathrm{Im}$ Verlag war man über «Thomas Manns Geschwätz» verärgert. Es sei merkwürdig, schrieb August Albers an einen ihm gut bekannten Schriftsteller, «dass ein Kerl wie Thomas Mann, der den Zauberberg schreiben konnte», sich herabwürdige, solchen «Quatsch» zu Papier zu bringen. Er tue es jedenfalls nur des Geldes wegen. ${ }^{39}$ In der Tat erzielte der Band keine hohen Absatzzahlen. Eine zweite Auflage erschien erst 1956, und Baeumlers monströse Vorrede wurde 1965 separat und mit einem Nachwort als «Sonderausgabe» unter dem Titel «Das mythische Zeitalter. Bachofens romantische Deutung des Altertums» publiziert. Heinrich Beck hatte schon Ende der zwanziger Jahre, allerdings vergeblich, angeregt, Einleitung und Edition separat zu verlegen, um den stagnierenden Absatz des Buches anzukurbeln. ${ }^{40}$

Auch sonst öffnete sich C.H.Beck in den zwanziger Jahren neuen Fragestellungen in den Altertumswissenschaften. Eine neue Generation suchte nach neuen Ansätzen, die den Historismus in den altertumskundlichen Disziplinen überwinden und die Kluft zwischen Wissenschaft und Leben schließen sollten. Sie warf der traditionellen Philologie vor, über Textkritik, Mikroskopie des Einzelnen und dem Aufsuchen von Beziehungen versäumt zu haben, nach dem Ganzen eines «Werkes» und seiner «Gestalt» zu fragen. Diese Philologen verehrten zwar Wilamowitz, lasen aber dennoch Nietzsche, Burckhardt und George. ${ }^{41}$ Der bedeutendste unter ihnen, der zu C.H.Beck fand, war Karl Reinhardt, der sein Poseidonios-Buch 1921 ebenso in München veröffentlichte wie 1926 sein Werk «Kosmos und Sympathie», das weitere Untersuchungen zu demselben hellenistischen Autor versammelte.

$\mathrm{Zu}$ den altertumswissenschaftlichen Publikationen traten Dichterbiographien: 1931 erschien «Gotthelf. Die Geheimnisse des Erzählers» des jungen Schweizer Literaturhistorikers Walter Muschg. Karl Vossler, einer der berühmtesten Romanisten der Zeit, veröffentlichte $193^{2}$ sein Buch «Lope de Vega und sein Zeitalter». Aber auch die Nationalökonomie und die neue Wissenschaft der Soziologie fanden Berücksichtigung: Im Jahr 
der Weltwirtschaftskrise publizierte der Volkswirt und Unternehmer Ludwig Reiners den ersten Band seines Buches «Die wirkliche Wirtschaft» (1930/33). Ebenfalls 1930 erschien die deutsche Bearbeitung der «Soziologischen Theorien im 19. und 2o. Jahrhundert», die Pitrim A. Sorokin, Professor an der Harvard University, verfasst hatte. Während Ludwig Reiners noch zahlreiche weitere Bücher zu unterschiedlichsten Themengebieten für C.H.Beck schrieb, blieb die Verbindung zu dem russischen Emigranten und Soziologen Episode. Verstärkt öffnete man sich der Religionswissenschaft und trug damit dem steigenden öffentlichen Interesse an dieser Disziplin Rechnung. So übernahm C.H.Beck das gesamte Werk des renommierten Marburger Religionswissenschaftlers Rudolf Otto. Sein epochemachendes Buch «Das Heilige. Über das Irrationale in der Idee des Göttlichen und sein Verhältnis zum Rationalen» kam $193^{1}$ in München heraus und ist noch heute bei C.H.Beck lieferbar. Auch Musikwissenschaft und Psychologie fehlten nicht: Der Geigenlehrer und Musikpädagoge Siegfried Eberhardt wollte mit seinem Buch «Der Körper in Form und in Hemmung. Die Beherrschung der Disposition als Lebensgrundlage» (1926) «Spitzenleistungen jeder Art mit der Haltung des Körpers in Parallele und ursächliche Beziehung setzen».22 Zwischen 1926 und 1939 erschienen darüber hinaus die «Neuen Psychologischen Studien», die in 15 Bänden von Felix Krueger herausgegeben wurden. Zu seinem Mitarbeiterkreis zählte Hans Volkelt, der Sohn von Heinrich Becks Leipziger Lehrer, der ein Freund des Verlegers seit Jugendtagen war. ${ }^{43}$ Schließlich entstand eine kleine Bibliothek mit Übersetzungen skandinavischer Romane, ${ }^{44}$ die auch die populären Werke von Johan Bojer, einem norwegischen Schriftsteller, umfasste, der in schlichter Sprache die Natur seiner Heimat und das Leben seiner Landsleute beschrieb. Dieses Projekt spiegelte die Faszination der Zeit an den Literaturen des Nordens, die vor allem in konservativen und völkischen Kreisen gepflegt wurde. ${ }^{45}$

\section{Die «Zeitwende»}

Heinrich Beck versuchte, «nach der Sanierung der Währung», allerdings «noch als unerfahrener Verleger», eine kulturpolitische Zeitschrift herauszubringen, die «Zeitwende» hieß und die im protestantisch-konservativen Milieu Bayerns verankert war. Als Vorbild diente möglicherweise die vielbeachtete Monatsschrift «Die Tat», die im Eugen Diederichs Verlag erschien. ${ }^{46}$ Als Herausgeber zeichneten Tim Klein, ein bekannter Feuilletonist der 
«Münchner Neuesten Nachrichten», Friedrich Langenfaß, Dekan der Evangelisch-Lutherischen Kirche in München, und Otto Gründler, ein im Zeitschriftenwesen erfahrener Schriftleiter, verantwortlich. ${ }^{47}$ Gründler war mit Karl Wolfskehl befreundet, allerdings wurde das gute Verhältnis durch Dissonanzen um Wolfskehls Veröffentlichungen in der Zeitschrift belastet. ${ }^{48}$

Über die Motive, die zur Gründung der Zeitschrift führten, gibt ein Prospekt aus dem Jahr 1924, Aufschluss. Durch die Katastrophen des letzten Jahrzehnts seien dem deutschen Volk, «ja der abendländischen Menschheit die Fundamente ihres scheinbar unzerstörbaren Kulturbesitzes erschüttert worden». So sei «an die Stelle des Gefühls der Selbstsicherheit die Sehnsucht nach objektivem Stand und Halt getreten». Der Name war programmatisch: «Von dieser geistigen Wende hat unsere Zeitschrift den Namen empfangen.» Diese Wende bedeute aber mehr als «eine reine zeitliche Umkehr». Sie dränge den Menschen, «bewusst wiederum das Ewige» zu suchen. Von dieser zu allen Zeiten «nötigen und wesentlichen Wendung der Zeit zur Ewigkeit soll der Name «Zeitwende` zeugen». Nach der Katastrophe des Ersten Weltkriegs sollte «das Kulturgewissen wieder mit Verantwortungsgefühl verbunden» und die «zentrale Stellung des reformatorischen Christentums» festgeschrieben werden. ${ }^{49}$

Am 1.Januar 1925 erschien die erste Nummer. Die Mitarbeiter waren fast durchweg protestantische Universitätstheologen, Pfarrer und Kirchenmänner. Jedes Heft bestand aus sechs bis sieben Artikeln, es folgten aktuelle «Umschau»-Texte und allerlei Randbemerkungen. Erzählungen, Gedichte und auch Fortsetzungsromane traten hinzu, wie etwa 1927 «Die Auswanderer» von Johan Bojer. «Bildbeilagen» und Fotografien lockerten den Satz auf. Religiöse Fragen standen im Vordergrund, aber einzelne Beiträge behandelten auch außen- und innenpolitische Zusammenhänge, gesellschaftliche und wirtschaftliche Entwicklungen, Philosophie und Psychologie, Musik, Theater, Literatur und Architektur. Die Heroen der deutschen Geschichte waren omnipräsent: Luther, Goethe, Nietzsche und Bismarck.

Die Themen waren mithin breit gefächert, oder genauer: die Programmlosigkeit war das Programm. ${ }^{50}$ Die Monatszeitschrift sollte mit dazu beitragen, C.H.Beck zu einem «Kulturverlag» des protestantischen Bürgertums zu machen. Der politische Kurs der Zeitschrift war konservativ. Die «Krisis der Demokratie» wurde häufig apostrophiert, die «Parteibureaukratie» attackiert und der «Selbstregierung des Volkes» applaudiert. ${ }^{51}$ Die Zeitschrift bezeichnete die «Judenfrage» als «eine der wichtigsten Fragen» der Zeit und verbreitete durchaus antisemitische Stereotype.52 Dennoch unterstützte sie nicht den nationalsozialistischen Rassenantisemitismus. Bruno 
Altmann etwa stellte die Frage nach der Existenz eines «Internationalen Faschismus» und berichtete über die Begegnung der italienischen Faschisten mit den deutschen Nationalsozialisten. Mussolini habe die Abgesandten des «Herrn Hitler» überhaupt nicht empfangen. Sein Staatssekretär Aldo Finzi habe ihnen jedoch Audienz gewährt. «Als sie aber gar so viel von arischer Hochwertigkeit und von semitischer Minderwertigkeit redeten, unterbrach sie Finzi mit dem Einwurf: Entschuldigen Sie, meine Herren, ich gehöre selbst zu dieser minderwertigen semitischen Rasse. Ich kann aber deshalb nicht so unglücklich sein, wie Sie es wollen, denn meiner Überzeugung nach dürften die beiden, der Arier wie der Semit, so ungefähr von demselben Affen abstammen.» ${ }^{53}$ Den älteren Lesern wurden auf sicherem lutherischen Fundament aktuelle Phänomene wie die Jugendbewegung erklärt: Der Erlanger Klassische Philologe und Pfarrerssohn Otto Stählin erklärte sie kurzerhand zu einer religiösen Bewegung, die deshalb so mächtig geworden sei, da die «neue Jugend» keine «Befriedigung in der verstandesmäßigen Klarheit der Kirchenlehre» gefunden habe. Aus ihr spreche ein «starkes, oft elementar hervorbrechendes Verlangen nach Gemeinschaft», das «soviele Leute der Jugendbewegung» eben doch «zur Christengemeinschaft» ziehe, um dort «eine neue Kultur aufzubauen». ${ }^{54}$

Während vor 1933 kritische Stimmen gegen den Nationalsozialismus Gehör fanden, erschienen nach der sogenannten Machtergreifung Beiträge, die den Systemwechsel begrüßten und Adolf Hitler als den gottgesandten Retter priesen, der Deutschland vor dem Bolschewismus bewahrt habe. Der schwedische Schriftsteller und Nazisympathisant Fredrik Böök erklärte, man müsse «sich unbedingt positiv zum Kampf Adolf Hitlers und der deutschen Jugend stellen. Es ist, könnte man sagen, der letzte, verzweifelte Versuch, Lebens- und Verjüngungskräfte bei einem Volk zu wecken, das nahe daran war, zu Tode gequält zu werden.» ${ }^{55}$ Offen wurde den Bücherverbrennungen Beifall bekundet. Im Lutherjahr 1933 lehnte der Herausgeber Otto Gründler die Republik von Weimar als Staat «offener Glaubensverhöhnung und Gotteslästerung» ab und bekannte sich zum neuen Staat, der «die ungeheure seelische und sittliche Bedeutung» der protestantischen Kirche «für den Aufbau einer wahren Volksgemeinschaft» erkannt habe. ${ }^{56}$ Die «Zeitwende» schwenkte auf die Richtung der Deutschen Christen ein. Zum zehnten Jubiläum der Zeitschrift im Jahre 1934 bekundeten die Herausgeber: «Wenn heute der nationalsozialistische Staat die christlichen Kirchen aufruft, an der sittlichen Erneuerung unseres Volkes mitzuarbeiten, wenn die geeinte Deutsche Evangelische Kirche das große 
Werk einer umfassenden Volksmission in Angriff nimmt, so können wir ohne Überheblichkeit sagen: in diesem Sinne arbeiten wir schon seit neun Jahren.» $^{57}$

Doch zu diesem Zeitpunkt war bereits offenkundig, dass sich der Verlag von der Monatsschrift trennen würde. Heinrich Beck empfand sie, so erklärte er später, als «Fremdkörper», deren protestantische Richtung seinen eigenen Anschauungen von Anfang an nicht entsprochen habe. Doch entscheidend dürfte gewesen sein, dass der Absatz rückläufig und die «Zeitwende auf finanzielle Zuschüsse» angewiesen war. ${ }^{58}$ Energisch warben die Herausgeber damals um neue Leser. ${ }^{59}$ Heinrich Beck trennte sich aus wirtschaftlichen Gründen von dem Periodikum, und seit Oktober 1934, erschien sie im evangelischen Wichern-Verlag in Stuttgart. Wenige Wochen zuvor hatte die neue «Deutsche Reichskirche» unter Bischof Ludwig Müller ein «Kirchengesetz über die Evangelische Presse» beschlossen, das die protestantische Presse gleichschalten sollte. Die absehbaren politischen Schwierigkeiten dürften Heinrich Beck bestärkt haben, sich von der Monatsschrift zu trennen. ${ }^{60}$

\section{Wechselfälle}

Die Entwicklung des juristischen Verlagsteils verlief in der Weimarer Republik weniger erfolgreich als im Kaiserreich. Nach dem Ende des Krieges begünstigte die auf Berlin ausgerichtete Verwaltung des Reiches die in der Reichshauptstadt angesiedelten Verlage. In der Weimarer Zeit verlor das bayerische Recht an Bedeutung. Die Beamten aus den Reichsministerien kamen häufig aus dem preußischen Staatsdienst. Der bayerische Verlag hatte es nicht leicht, unter ihnen gute Autoren zu gewinnen. Zudem wirkte es sich nachteilig aus, dass im Verlag keine juristische Zeitschrift herausgegeben wurde und kein Jurist als Lektor tätig war. Allmählich verschwanden die im Kaiserreich bekannten und beliebten Textausgaben und die mittelgroßen «Handausgaben», darunter auch die des BGB. Das «Flaggschiff» der Handausgaben erschien zuletzt in 13. Auflage 1927. Ein Lichtstreifen am Horizont zeigte sich, als Heinrich Schönfelder, später Amtsgerichtsrat in Dresden, die Reihe «Prüfe dein Wissen» für die juristische Ausbildung herausgab, die bis 1933 alle maßgeblichen Rechtsgebiete erschloss. Schönfelder regte die Reihe «Deutsche Reichsgesetze» an, die die wichtigsten Gesetze zu einem günstigen Preis enthalten sollte. Das Format wurde auf Anhieb ein großer Erfolg und zählte zu den stärksten und langlebigsten 
Marken des Hauses. Doch im juristischen Bereich kam es erst durch die Übernahme des jüdischen Verlags Otto Liebmann, der in Berlin angesiedelt war, zu einem deutlichen Aufwärtstrend. ${ }^{61}$

Auf dem umkämpften Büchermarkt wurde für alle Veröffentlichungen intensiv geworben. Verantwortlich hierfür war die Abteilung für «Propaganda», die von denselben Mitarbeitern getragen wurde, die auch Lektoratsaufgaben versahen und für die Autorenakquisition und -betreuung zuständig waren. Jubiläen wurden zunehmend wichtig. August Albers verwandte sich Anfang September 1929 für das Kleistbuch des konservativen katholischen Philosophen und Theologen Carl Braig, das bereits 1925 bei C.H.Beck erschienen war, aber «seitens der für Christentum unempfindlichen Universitätsprofessoren sozusagen den Genickfang bekommen» habe. Albers bat Hans Ludwig Held, den Direktor der Münchner Stadtbibliothek, die Biographie aus Anlass der 15o. Wiederkehr von Kleists Geburtstag in einer Zeitschrift anzuzeigen. ${ }^{62}$ Wenn Zeitschriften die zugesandten Bücher nicht besprachen, hakte der Verlag nach; bisweilen sanktionierte man unbotmäßiges Verhalten dadurch, dass keine neuen Rezensionsexemplare versandt wurden. Albers ließ 1934, Rudolf Bultmann, den Schriftleiter der «Theologischen Rundschau», wissen, dass von Rudolf Ottos «Reich Gottes und Menschensohn» leider keine Besprechungsexemplare abgegeben werden könnten; denn in der «Kartothek» des Verlags stünden noch fünf Bücher, die der Verlag an die Zeitschrift geschickt habe, die aber nie angezeigt worden seien. Mahnend fügte er hinzu: «Es sind Werke, die bis ins Jahr 1929 zurückreichen.» ${ }^{63}$

Trotz Werbung gingen manche Titel einfach schlecht: Von Johann Jakob Bachofens «Der Mythus von Orient und Occident. Eine Metaphysik der alten Welt» hatte der Verlag 1926, wie August Albers klagte, 70 Rezensionsexemplare versandt, die aber nur auf geringes Interesse gestoßen waren. ${ }^{64}$ Doch der Blick auf die Titelproduktion und die Bilanzen zeigt, dass trotz der zeitbedingten Schwierigkeiten die unternehmerische Ausrichtung des Verlags in den zwanziger Jahren erfolgreich war. C.H.Beck gehörte nicht zu den ganz Großen der Branche wie etwa Julius Springer in Berlin oder B. G. Teubner in Leipzig, aber gleichwohl war das Verlagshaus in der Weimarer Republik einer der führenden Verlage: 1920 erschienen 85 neue Titel (zum Vergleich J. Springer: 386 / B. G. Teubner: 388), 1922106 (462 / 345) und 192792 (436 / 590). Vor dem Krieg, genau: 1913, hatte die Titelproduktion 91 ( $379 / 628)$ betragen. ${ }^{65}$ Mithin hatte der traditionsreiche Verlag nach dem Krieg nur einen mäßigen Einbruch erlebt und konnte im Gegensatz zu anderen Unternehmen - wie etwa Carl Winters Universitäts- 
buchhandlung in Heidelberg - das Produktionsvolumen rasch wieder stabilisieren. Mit diesem Befund korrespondiert die Bilanzentwicklung. Die erste Bilanz nach den Wirren der Hyperinflation im Jahr 1924, belief sich auf 728113,07 RM. ${ }^{66}$ Die folgenden Bilanzen bestätigen die ökonomische Konsolidierung des Unternehmens in der zweiten Hälfte der zwanziger Jahre. ${ }^{67}$ Für 1925 wurde eine Bilanzsumme von 8o6883,56 RM ausgewiesen, der Reingewinn belief sich auf 45336,411 RM, für 1926809 177,82 RM (4,8 983,49 RM), für 1927919277,71 RM (67 030,70 RM) und für 1928922960,47 RM (101 971,79 RM). 1929 wurde die Millionengrenze überschritten: Die Bilanzsumme betrug 1 4,18 4,45,27 RM, der Reingewinn 176 o9o,88 RM. ${ }^{68}$

In diesem Jahr des wirtschaftlichen Erfolgs heiratete der Verleger die 23-jährige Eva Müller, eine Tochter des Ministerialdirektors Ernst von Müller, der im bayerischen Ministerium des Äußeren tätig war. Zur Hochzeit am 8. Mai ließen Karl Wolfskehl und Otto Scheiner von der RupprechtPresse «Der Liebe Wechselgesang» aus Goethes «West-östlichem Divan» ertönen. ${ }^{69}$ Aus der Ehe gingen zwei Söhne und drei Töchter hervor: Veronika, geboren am 10. Juni 1930, Hans Dieter, geboren am 9. April 1932, Liselotte, geboren am 24. Dezember 1934, Susanne, geboren am 14. Juli 1937, und Wolfgang, geboren am 29. September 1941.

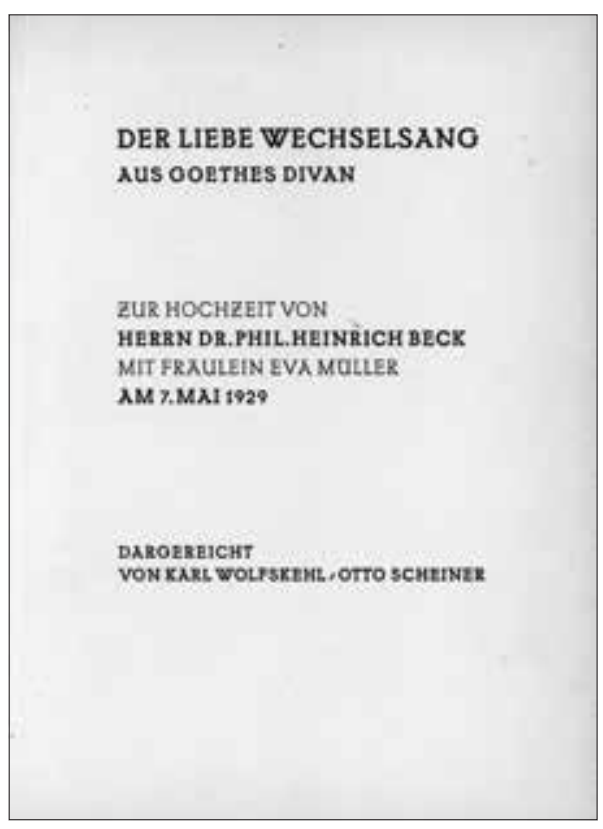

Hochzeitsgabe für den jungen Verleger Heinrich Beck und seine Frau Eva Beck, geb. Müller
Die wirtschaftliche Entwicklung von C.H.Beck spiegelt die Gesamtsituation im Verlagswesen. Nach dem Ende des Ersten Weltkriegs kam es zu einem erst zögerlichen, dann deutlichen Aufschwung, der auf der Neuauflagenproduktion basierte, so dass sich wirtschaftlich keineswegs eine «Bücherkrise» feststellen lässt. Infolge der weltwirtschaftlichen Turbulenzen nach 1929 nahm das Produktionsvolumen jedoch wieder deutlich ab. ${ }^{70}$ Hatte sich die Bilanzsumme des Verlags C.H.Beck für 1931 noch auf 1 313 324,70 RM belaufen und sich nur minimal gegenüber 1930 verändert (1 413030,31 RM), so halbierte sich der Gewinn auf 51529,33 RM (1930: 100644,38 RM). Die Bilanzsumme für $193^{2}$ fiel auf 94,1 495,86 RM (20 147,81 RM) und für 1933 auf 882 4,25,40 RM $(24,277,14, \mathrm{RM}){ }^{71}$

Die Vermögenssituation erlaubte es dem 
Verleger, seinen gesellschaftlichen Pflichten nachzukommen. Dazu zählte auch, dass er seine Autoren einlud. In seinem Haus verkehrten Oswald Spengler und Egon Friedell, und bisweilen schaute auch Albert Schweitzer vorbei. $^{72}$ Allerdings wurde das Haus in der Kunigundenstraße, das er seit 1929 bewohnte, nicht zum großbürgerlichen Treffpunkt von Intellektuellen. Die glanzvolle Ausstrahlung, die der Salon des Münchner Verlegerehepaars Hugo und Elsa Bruckmann vor und nach dem Ersten Weltkrieg entfaltete ${ }^{73}$ wollte Heinrich Beck nicht imitieren: Dafür waren die Mittel, die er für solche Zusammenkünfte aufzuwenden bereit war, zu gering. Übermäßiger Luxus lag ihm ohnehin fern; er bevorzugte eher einen bescheidenen Habitus. In der Bruckmann'schen Villa verkehrten allerdings nicht nur Wissenschaftler, Künstler und Schriftsteller; dort wurde auch der junge Adolf Hitler in die feine Münchner Gesellschaft eingeführt. ${ }^{74}$

Mit den Nationalsozialisten aber wollte der konservative Verleger Heinrich Beck zunächst nichts zu tun haben. Politisch stand er vielmehr auf der Seite des elitären Oswald Spengler. Sonst wäre es ihm unmöglich gewesen, den «Untergang des Abendlandes» zu verlegen, wie er später offen einräumte. ${ }^{75}$ Individualistischen Liberalismus und materialistischen Kapitalismus lehnte der junge Verleger ab. Vor Bolschewismus und Revolution grauste ihm. In der von politischen Konvulsionen traumatisierten Weimarer Republik favorisierte Heinrich Beck den Intellektuellen der Konservativen Revolution, der gegen demokratische und parlamentarische Strömungen Position bezog und die emanzipatorische Tradition der Aufklärung verwarf. Diese politisch-ideologische Position spiegelte die biographische Erfahrung. Heinrich Beck war 1914, 25-jährig begeistert ins Feld gezogen und konnte sich nach 1918 mit der Niederlage nicht abfinden. Bezeichnend ist seine Beurteilung des Soldaten Walter Flex, dem er sich geistesverwandt fühlte: «Nun sollten die ethischen Voraussetzungen, die eine Elite der deutschen Jugend in sich geformt hatte, im Krieg erhärtet werden», schrieb Heinrich Beck noch 1963. Der nur zwei Jahre ältere Flex repräsentierte für ihn eine «Geistesepoche, in der die deutsche Jugend sich ihres Wertes bewusst wurde und die Bevormundung der älteren Generation abstreifte, in edler und überzeugender Weise». ${ }^{76}$ Heinrich Beck streifte die Bevormundung durch seinen Vater ab und führte den Verlag zu neuen Ufern. Der Geist des wilhelminischen Zeitalters sollte nicht weiter die Verlagspolitik bestimmen.

Durch sein Programm wollte Heinrich Beck dem suchenden Bürgertum in Zeiten der wirtschaftlichen und politischen, der weltanschaulichen und emotionalen Instabilität Orientierung geben. Dabei war er selbst ein 
Suchender. Als solcher besuchte er die «Lauensteiner Tafelrunden», deren Teilnehmer sich auf der Burg Lauenstein zwischen Thüringer und Frankenwald trafen. Die Initiative hatte Eugen Diederichs ergriffen, der eine neue «Geisteskultur» begründen wollte, auf Reformen im Buchhandel drängte und die jüngere Verlegergeneration zu kulturpolitischen Diskussionen versammelte. ${ }^{77}$ Mit dem Jenenser Verleger schloss Heinrich Beck auf den Lauensteiner Tagungen Freundschaft. ${ }^{78}$ Stand Heinrich Beck auch politisch rechts, so öffnete er den Verlag dennoch nicht nur Autoren der antiparlamentarischen Opposition und der Konservativen Revolution. Die Marktkonkurrenz verbot eine solche Beschränkung. Als Unternehmer war er vielmehr bestrebt, kulturpolitische Intentionen mit kaufmännischem Kalkül zu verbinden. Mit Hilfe seiner beiden engsten Mitarbeiter August Albers und Walther Eggert-Windegg machte er C.H.Beck nach der Katastrophe des Ersten Weltkriegs und inmitten der ungeliebten Republik zu einem Experimentierfeld der intellektuellen Avantgarde. 